\title{
EFFECTS OF SHUTTER TIMING ON CCD PHOTOMETRY
}

\author{
D. Galadí-Enríquez, C. Jordi and E. Trullols \\ Dept. d'Astronomia i Meteorologia, Universitat de Barcelona
}

CCD-shutters take some time to open and close. This results in a difference between the recorded and real exposure times. The pixel-to-pixel pattern $\delta(x, y)$ in which this difference varies depends on the characteristics of the device. These timing errors affect photometric measurements in two ways: directly, since the recorded exposure time is not the real one, and indirectly, via the flatfielding process, because the shutter effect is mixed up with the flatfield pattern. The direct effect may be negligible when exposure times are long. The indirect effect is present irrespective of the exposure times of the frames, if flatfield exposure times are short.

Generalizing Stetson (1989), to calculate the function $\delta(\mathrm{x}, \mathrm{y})$ we take a set of $\mathrm{n}$ flatfields, $\mathrm{f}_{\mathrm{i}}(\mathrm{x}, \mathrm{y})$, with recorded exposure times $\operatorname{tau}_{\mathrm{i}} \mathrm{n}$ and another flatfield, $\mathrm{F}(\mathrm{x}, \mathrm{y})$, with recorded exposure time $\mathrm{T}$, all of them obtained under the same illumination conditions. Defining the ratio:

$$
R(x, y)=\frac{\sum_{i=1}^{n} f_{i}(x, y)}{F(x, y)}
$$

we can compute the shutter map as

$$
\delta(x, y)=\frac{R(x, y) T-\sum_{i=1}^{n} \tau_{i}}{n-R(x, y)}
$$

Shutter effects can be removed from any given image $Z(x, y)$ with recorded exposure time $t_{n}$, by performing the calculation

$$
\hat{Z}(x, y)=Z(x, y) \frac{t_{n}}{t_{n}+\delta(x, y)}
$$

which yields the corrected image $\hat{Z}(x, y)$. Another approach to shutter-timing errors was proposed by Surma (1993).

Our study of the CCD-shutters on the 1.23-m and 2.2-m telescopes of CAHA at Calar Alto (Almeria, Spain), shows that shutter-timing influence is not negligible. For a $10 \mathrm{~s}$ exposure, $\delta(\mathrm{x}, \mathrm{y})$ yields a difference of $0.010 \mathrm{mag}$ between the most and less exposed pixels, and a global error of $0.037 \mathrm{mag}$ due to the general over-exposure of all the frame.

\section{REFERENCES}

Stetson, P. B. 1989 Highlights of Astronomy 8, 635

Surma, P. 1993 A\&A 278, 654 